\title{
Low Fraction Size Re-irradiation for Large Volume Recurrence of Glial Tumours
}

\author{
Ágnes Dobi ${ }^{1}$ - Barbara Darázs ${ }^{1} \cdot$ Emese Fodor $^{1} \cdot$ Adrienne Cserháti $^{1} \cdot$ Zsófia Együd $^{1} \cdot$ Anikó Maráz $^{1} \cdot$ Szilvia László $^{1}$. \\ Leopold Dodd ${ }^{1} \cdot$ Zita Reisz $^{2} \cdot$ Pál Barzó $^{3} \cdot$ Judit Oláh $^{1} \cdot$ Katalin Hideghéty ${ }^{1}$
}

Received: 29 April 2020 / Accepted: 30 June 2020 / Published online: 9 July 2020

(C) The Author(s) 2020

\begin{abstract}
The aim of the present study was to evaluate the efficacy of re-irradiation (re-RT) in patients with advanced local relapses of glial tumours and to define the factors influencing the result of the hyper-fractionated external beam therapy on progression after primary management. We have analysed the data of 55 patients with brain tumours (GBM: 28) on progression, who were reirradiated between January 2007 and December 2018. The mean volume of the recurrent tumour was $118 \mathrm{~cm}^{3}$, and the mean planning target volume (PTV) was $316 \mathrm{~cm}^{3}$, to which $32 \mathrm{~Gy}$ was delivered in 20 fractions at least 7.7 months after the first radiotherapy, using 3D conformal radiotherapy (CRT) or intensity modulated radiotherapy (IMRT). The median overall survival (mOS) from the re-RT was 8.4 months, and the 6-month and the 12-month OS rate was 64\% and 31\%, respectively. The most important factors by univariate analysis, which significantly improved the outcome of re-RT were the longer time interval between the diagnosis and second radiotherapy $(p=0.029)$, the lower histology grade $(p=0.034)$, volume of the recurrent tumour $(p=0.006)$ and Karnofsky performance status (KPS) $(p=0.009)$ at the re-irradiation. Our low fraction size re-irradiation $\geq 8$ months after the first radiotherapy proved to be safe and beneficial for patients with large volume recurrent glial tumours.
\end{abstract}

Keywords Re-irradiation $\cdot$ Multiform glioblastoma $\cdot$ Glioma

\section{Introduction}

Gliomas, with incidence of 5/100 000 in adults, are the most common primary central nervous system malignancies, peaking between the fifth and sixth decades of life [1]. After initial multimodal treatment, at least $70 \%$ recurrence rate of gliomas can be expected [2-4]. By surgical therapy alone, the disease has a very poor prognosis (median survival 4-6 months [5], whereas surgery accompanied by radiotherapy (RT) ameliorates the median survival data to 8-9 months. Together with concomitant and sequential TMZ, better median survival values can be expected,

Ágnes Dobi

dobiagnes@gmail.com

1 Department of Oncotherapy, University of Szeged, Korányi fasor 12, Szeged H-6720, Hungary

2 Department of Pathology, University of Szeged, Állomás utca 1, Szeged H-6725, Hungary

3 Department of Neurosurgery, University of Szeged, Semmelweis utca 6, Szeged H-6725, Hungary such as 15 months for glioblastomas, or even 2-5 years for anaplastic gliomas [6].

In the case of recurrence with its considerable limitations, and only if it is possible, surgical treatment has the highest efficacy [7]. In certain good performance status patients with good anatomical access to tumours, surgery is applicable, but the resection outcome could be definitely limited by considerable infiltration of nervous tissue and by higher morbidity risk [8,9]. As for other low grade and grade 3 cases, temozolamide (TMZ) is the treatment of choice, if it was not administered during the initial management. Thereafter and for GBM second-line systemic treatment (such as chemo- or biological therapy) and re-irradiation is optional, in the lack of standardised treatment for recurrent gliomas [10]. Recently, Tumour Treating Field (TTF), a novel therapeutic option emerged prolonging the survival with further 6 months [1]. For systemic treatment, monoclonal antibody (bevacizumab), chemotherapy (nitrosurea, lomustine, dose dense TMZ [9], immune checkpoint blockade (nivolumab, pembrolizumab) [11], or even vaccines (DCVax) [12-14] are options to consider. For recurrent tumours, salvage re-irradiation could be selected. The typical re-irradiation techniques and strategies 
for recurrent gliomas are conventionally fractionated RT, brachytherapy, hypofractionated stereotactic radiosurgery (FSRT), stereotactic radiosurgery (SRS) alone, or combination treatment with RT and systemic therapy, and palliative RT $[15,16]$.

On reviewing several clinical trials, the 6- and 12-month overall survival (OS-6 and OS-12), calculated from the time of re-irradiation, were $73 \%$ and $36 \%$, respectively, whereas the 6- and 12-month of progression free survival (PFS-6 and PFS12) were $43 \%$ and $17 \%$, respectively [17]. Median OS (mOS) was 7.4-12.7 months in other studies [18-22].

\section{Materials and Methods}

Between 2007 and 2018, at the Department of Oncotherapy, altogether 55 patients with recurrent glial tumours were subjected to re-irradiation. The present study has been carried out in accordance with The Code of Ethics of the World Medical Association (Declaration of Helsinki) for experiments involving humans. Informed consent was obtained from the patients at their first clinical admission for the anonymised use of their patient data for research purposes. According to Sect. 20/Q of No. 23/2002 Decree of the Ministry of Health, Hungary, the present study is considered as a non-interventional clinical study. The whole present study was carried out according to the ethical permission No. 4209/2018-SZTE, issued by the Ethical Committee of our University. The treatment schemes were thoroughly discussed with every single patient, independently from their actual performance status. The re-irradiation was agreed by signed informed consent. The initial care consisted of surgery in each case. The patients with grade 2 and grade 3 brain tumours received radiotherapy only postoperatively and for GBM we applied adjuvant chemoradiation therapy followed by temozolamide monotherapy up to progression. Magnetic resonance imaging (MRI) were performed three monthly. Disease progression was defined by two independent experts. At the time of diagnosis, the tumour grading was based on histological assessment. At the time of re-RT, histological evaluation was performed only in the re-operated cases, in the case of the remaining patients (without re-operation), the grading was based on clinical and radiological evaluation. The re-irradiation volume was defined on the basis of planning CT (computed tomography) and MRI fusion. Patients were immobilised with a 3-point thermoplastic mask (ORFIT Industries, NL). The planning target volume encompassed the GTV (gross tumour volume) plus $0.3-1 \mathrm{~cm}$ margin. The shapes of the recurrent tumours were frequently highly irregular, sometimes with multiple manifestations, and with spread to the contralateral hemisphere through the corpus callosum; or spreading along the wall of the previous surgical cavity and/or ventricle wall, resulting in larger PTV (planning target volume). The normal structures were contoured including the lens, optic chiasm, optic nerve, brain, and brainstem. Treatment planning was performed with Eclipse (version 5, Varian Medical Systems, Palo Alto, USA). The re-RT dose was 32 Gy in 1.6 Gy daily fractions in all cases, in order to avoid serious neurotoxicity. Dependent on the location and extent of the recurrent glioma, 3 DCRT or IMRT or VMAT (Rapid Arch) therapy-plans (VMAT) were generated according to the ICRU (International Commission on Radiation Units \& Measurements, Inc.) 52 recommendation. [23].

During brain irradiation, patients received $12 \mathrm{mg}$ methylprednisolone for prevention of brain oedema, with gradually decreased dosing after radiotherapy. The dose of methylprednisolone was adjusted according to the symptoms of intracranial pressure elevation due to brain oedema. The majority of the patients ( 32 over 23 ) received bevacizumab therapy after the re-RT, and these patients were controlled in a biweekly fashion, with physical examination up to progression and 3-months intervals MRIs were performed, whereas for the remaining group without bevacizumab treatment after re-RT, the check-ups were scheduled in 4-6 weeks. Two experts evaluated the images according to the RANO HGG (Response Assessment in Neuro-Oncology High-grade glioma) criteria [24]. We included all patients with a recurrent glial tumour who completed the 32 Gy re-irradiation in 20 fractions to the present analysis. We assessed retrospectively the overall survival (OS) from the diagnosis, and from the first day of the re-irradiation according to the, age, Karnofsky performance score (KPS), primary tumour grade and histopathology type, the type of the primary tumour removal, size of GTV, size of PTV, time interval between two irradiations, time elapsed between diagnosis and 2nd RT, second line bevacizumab treatment. The data were evaluated by KaplanMeier statistical analysis with IBM SPSS Statistics for Windows, Version 20.0 (Armonk, NY: IBM Corp.) p value $<0.05$ was considered as statistically significant. COX regression was used for univariate, as well as multivariate analysis. Factors with significance in univariate analysis were included into a multivariate analysis. After the re-irradiation we recorded the KPS, Mini Mental Score (MMS) and daily activity in every visit.

\section{Results}

\subsection{Patient Characteristics}

Table 1 summarises the patient characteristics. The mean age of the population at the time of the primary diagnosis detection was 39 years (range: $11-71$ years); $49 \%$ of them was male and $51 \%$ female. The mean age at the time of the re-irradiation was 42 years (range: $13-72$ years). The KPS was in $40 \%$ of this population over $70 \%$. At the beginning of the reirradiation, 
Table 1 Summary of the patient characteristics

\begin{tabular}{ll}
\hline Variables & No. of the patients \\
\hline Number of the patients & 55 \\
Sex & \\
Male & 27 \\
Female & 28 \\
KPS & \\
$>70 \%$ & 22 \\
$\leq 70 \%$ & 33 \\
Primary histopathology type & \\
astrocytoma grade 2 & 15 \\
oligodendroglioma grade 3 & 6 \\
anaplastic astrocytoma grade 3 & 6 \\
glioblastoma multiforme & 28 \\
Salvage surgery & 23 \\
Prior temozolomid treatment & 55 \\
MGMT methylation status & \\
methylated & 18 \\
unmethylated & 9 \\
unknown & 28 \\
\hline
\end{tabular}

majority of the patients had minor neurological symptoms, such as hemiparesis, facial paresis, focal seizure partly controlled by antiepileptic medication, more frequently motor and sensory aphasia. Besides these symptoms, the patients preserved the ability of self-caring, except 5 patients with serious paresis needing regular help in their daily life. Out of 23 cases with repeated surgery, only four initially grade 2-3 tumours showed malignant transformation to grade $3-4$. In the majority of the cases, though, reoperation took place relatively early during the course of the disease. Usually in the case of initially low grade tumours, surgery was performed prior to the first oncological management; and also grade 3 tumours were as well as re-operated some years prior to reirradiation. In $84 \%$ of the cases, based on the clinical behaviour of the tumour, their malignant transformation was highly probable at the time of reirradiation, but no regular biopsy was performed in order to confirm it. The average time interval between the diagnosis and re-irradiation was 47.4 months (range: $7.3-228$ months) first and the re-irradiation was 36 months (range: 7.7232 months) respectively. All patients received first-line systemic temozolomide treatment, either as part of initial postoperative management (GBM), or at the first relapse. 23 patients were treated with bevacizumab monotherapy, as second-line treatment. The re-irradiation was performed after the first-line systemic treatment in 45 cases, and after second-line therapy in 10 cases. The primary histological type was grade 2 astrocytoma in 15 cases, grade 3 glial tumour (anaplastic astrocytoma or oligodendroglioma) in 12 cases, and glioblastoma multiforme (GBM) in 28 cases. MGMT (O-6-
methylguanine-DNA methyltransferase) methylation status was known in 26 cases. 16 patients were methylated, 9 patients borderline methylated and in one case MGMT was nonmethylated.

\subsection{Survival Analysis}

Median survival was altogether 42.6 months, as calculated from the date of the first diagnosis. The Table 2 shows the survival data. Regarding histology, cases with lower, grade 2 malignancies had the most favourable survival values (111.0 months), whereas this value was 23 months $(\mathrm{p}<0.001)$ in cases with GBM. We found a strong correlation to histological type: grade 2 astrocytoma cases had the longest survival (114.8 months), whereas the worst survival was detected of grade 4 cases $(30.7$ months; $\mathrm{p} \leq 0.001)$.

The most important factors significantly influencing the outcome of re-RT were the time interval between the first and second radiotherapy, histology grade, GTV, and KPS at the re-irradiation.

\subsection{Survival from the Beginning of Re-irradiation}

The mOS from the re-RT of the entire cohort was 8.4 months; 6 patients survived more than 10 months and 2 patients more than 2 years. The 6-month and the 12 -month OS rate was $64 \%$ and $31 \%$ respectively.

The mean volume of GTV, as contoured during Re-RT, was $118.0 \mathrm{~cm}^{3}$ (range: $4.5-304 \mathrm{~cm}^{3}$ ). Patients with lesser than average GTV at re-RT had 12.9 months, patients with greater than average GTV at re-RT had 5.5 month of median survival $(\mathrm{p}=0.006)$ (Fig. 1).

Patients with KPS $>70 \%$ at the beginning of re-RT had significantly better survival values (10.4 months, $p=0.009)$, than those ones with poorer general conditions (5.6 months) (Fig. 2).

Comparing time interval (between diagnosis and second radiotherapy, reRT) with OS on univariate analysis, patients with an interval of more than 47 months from 1 st to the 2 nd course of RT (mOS 10.2 vs. 6.7 months, hazard ratio (HR) $0.99,95 \%$ confidence interval (CI) $\mathrm{p}=0.029$. (Fig. 3).

Median survival, as calculated from re-irradiation for the entire group was 9.0 months. According to histopathology GBM: 6.0 months, grade $2+3$ malignancies 10.0 months; $\mathrm{p}=0.031$.

These factors proved to be significant for OS from re-RT in cox-regression univariate analysis. In multivariate analysis, the smaller GTV and better KPS remained significant influencing factors.

In the present study, no significant interrelation was found between OS and age at re-RT, size of PTV, type of primary surgical intervention, or whether the second-line bevacizumab therapy preceded or followed the re-irradiation. 
Table 2 Survival data. Significant correlations between investigated factors are highlighted with bold characters

\begin{tabular}{|c|c|c|c|c|}
\hline Variable & $\mathrm{n}$ & OS (months) & $\pm \mathrm{SE}$ & p-value \\
\hline & & from initial di & & \multirow{7}{*}{$\mathrm{p}<0.001$} \\
\hline Entire group & 55 & 42.6 & 2.6 & \\
\hline \multicolumn{5}{|l|}{ initial histopathology type } \\
\hline grade 2 & 15 & 114.8 & 40.2 & \\
\hline grade 3 & 12 & 52.2 & 9.8 & \\
\hline \multirow[t]{2}{*}{ grade 4} & 28 & 30.7 & 1.3 & \\
\hline & & \multicolumn{2}{|l|}{ from re-RT } & \\
\hline entire group & 55 & 8.37 & 1.9 & \\
\hline \multicolumn{5}{|l|}{ histopathology type at re-irradiation } \\
\hline $\operatorname{grade} 2(n=12)+\operatorname{grade} 3(n=14)$ & 26 & 10 & 1.2 & \multirow[t]{2}{*}{$p=0.031$} \\
\hline grade 4 & 29 & 6 & 2 & \\
\hline \multicolumn{5}{|l|}{ GTV re-RT mean $118 \mathrm{~cm}^{3}$} \\
\hline$\leq$ mean & 29 & 12.9 & 3.9 & \multirow[t]{2}{*}{$p=0.006$} \\
\hline$>$ mean & 23 & 5.5 & 0.3 & \\
\hline \multicolumn{5}{|l|}{ KPS at re-RT } \\
\hline$\leq 70 \%$ & 33 & 5.6 & 0.7 & \multirow[t]{2}{*}{$p=0.009$} \\
\hline$>70 \%$ & 22 & 10.4 & 1.9 & \\
\hline \multicolumn{5}{|c|}{ Time between diagnosis (DG) and re-RT } \\
\hline$\leq 47$ months & 18 & 6.7 & & \multirow[t]{2}{*}{$p=0.029$} \\
\hline$>47$ months & 37 & 10.2 & & \\
\hline \multicolumn{5}{|l|}{ PTV re-RT $316 \mathrm{~cm}^{3}$} \\
\hline$\leq$ mean & 33 & 10.1 & 1.5 & \multirow[t]{2}{*}{$\mathrm{p}=0.246$} \\
\hline$>$ mean & 22 & 5.5 & 0.4 & \\
\hline \multicolumn{5}{|l|}{ Age at re-irradiation } \\
\hline$\leq 40$ year & 27 & 8.3 & 2.2 & \multirow[t]{2}{*}{$\mathrm{p}=0.704$} \\
\hline$>40$ year & 28 & 6.6 & 2.7 & \\
\hline \multicolumn{5}{|l|}{ bevacizumab therapy before re-RT } \\
\hline no & 32 & 6.5 & 1.1 & \multirow[t]{2}{*}{$\mathrm{p}=0.35$} \\
\hline yes & 23 & 10.2 & 0.3 & \\
\hline
\end{tabular}

\subsection{Outcome and Toxicity of the Treatment}

Due to the second radiotherapy at progression, amelioration of neurological signs and KPS were experienced in $58 \%$ of our patients. Control radiological imaging detected stable disease or partial remission in 44 cases.

In the majority of the cases, the prophylactic dose and escalated dose on demand of the methyl-prednisolone prevented the development of serious brain oedema and the consequent intracranial pressure elevation. We did not see any radiation necrosis on the follow up MRIs and no major cognitive deficit was associated to the re-RT assessed with MMS.

\section{Discussion}

In the past, re-irradiation for recurrent malignant gliomas was considered with great reluctance due to the high risk of radiation necrosis. In the recent decades after implementation of advanced RT techniques, several retrospective analyses, reviews, as well a prospective study and meta-analysis were published proving that re-irradiation is useful treatment option for recurrent brain tumours [16, 17, 25, 26].

The present work represents the establishment of a careful, consequent re-RT approach with low fraction size to avoid radiation sequelae using standardised target volume and dose definition even for large volume recurrences. Selected studies on SRS for small volume recurrences of median 6.2 to $28.0 \mathrm{~cm}^{3}$ [27-29] showed an improvement on median survival ranging from 5.3 to 13.0 months with associated radionecrosis of $0-31.3 \%$. Others have reported their results for recurrent GBM volumes of median 7-50 $\mathrm{cm}^{3}$ applying FSRT resulted in median survival within the range of 6.5-11 months [26, 30-32]. In contrast to these small target volumes for re-RT with SRS techniques, lower doses to larger volumes could be applied safely with acceptable efficacy, which was confirmed by the first meta-analysis on re-irradiation published by Kazmi et al. [17]. However, highly divergent 


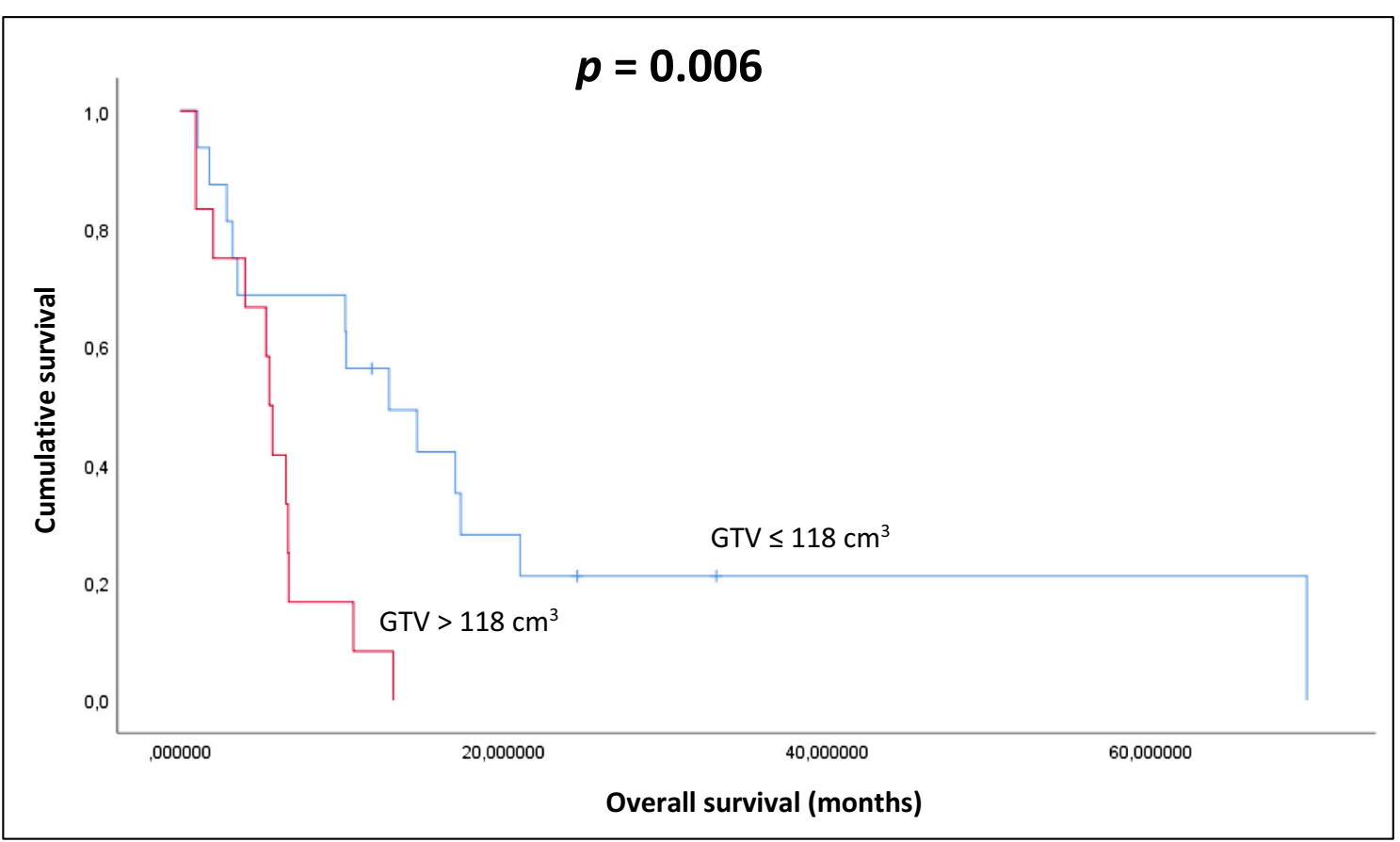

Fig. 1 Kaplan-Meier plot of the correlation between GTV-re-irradiation and OS ( $p=0.006)$

fractionation schemes and target volume concepts are applied with heterogeneous median survival times between 5 and 18 months. Krauze et al. reported a mOS of 6 months after reirradiation of recurrent glioma with median 30 Gy [33]. Another recent study revealed that OS after salvage SRS or hypofractionated RT (HFRT) does not significantly $(\mathrm{p}=0.06)$ differs from that after conventionally fractionated re-RT, and the trend towards better OS probably related to smaller target volume [34]. Analysis in a retrospective review has not shown any differences in OS after stereotactic or conventionally fractionated re-RT [35]. The similar outcome (mOS of 9.7 months) using conventional-, hypofractionated or SRS techniques was confirmed by another retrospective analysis of reRT for recurrent malignant glioma [36]. The 9-month mOS with re-RT achieved in our patients with GTV median of $118 \mathrm{~cm}^{3}$ falls within the range of previously reported series

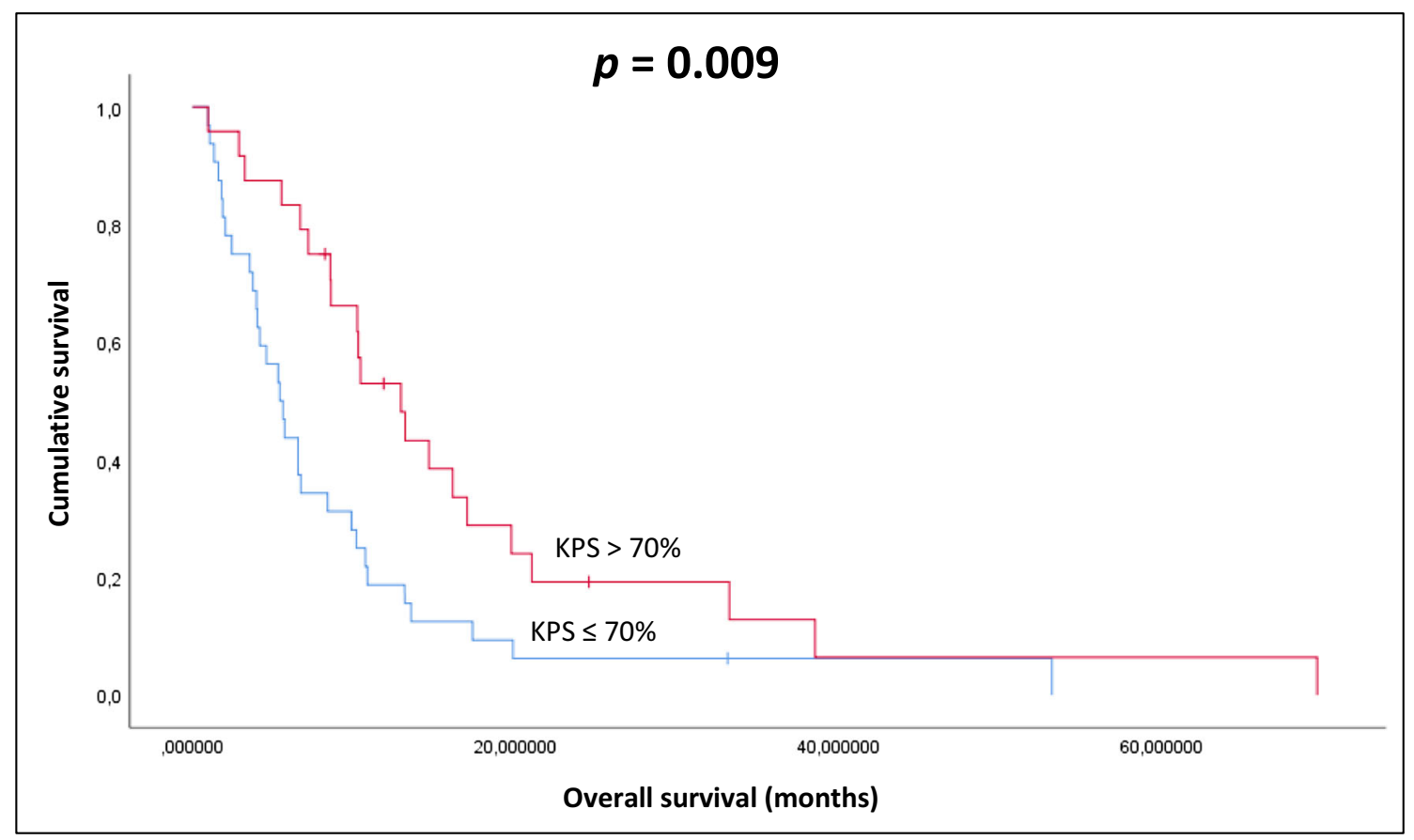

Fig. 2 Kaplan-Meier plot of the correlation between KPS and OS ( $\mathrm{p}=0.009)$ 


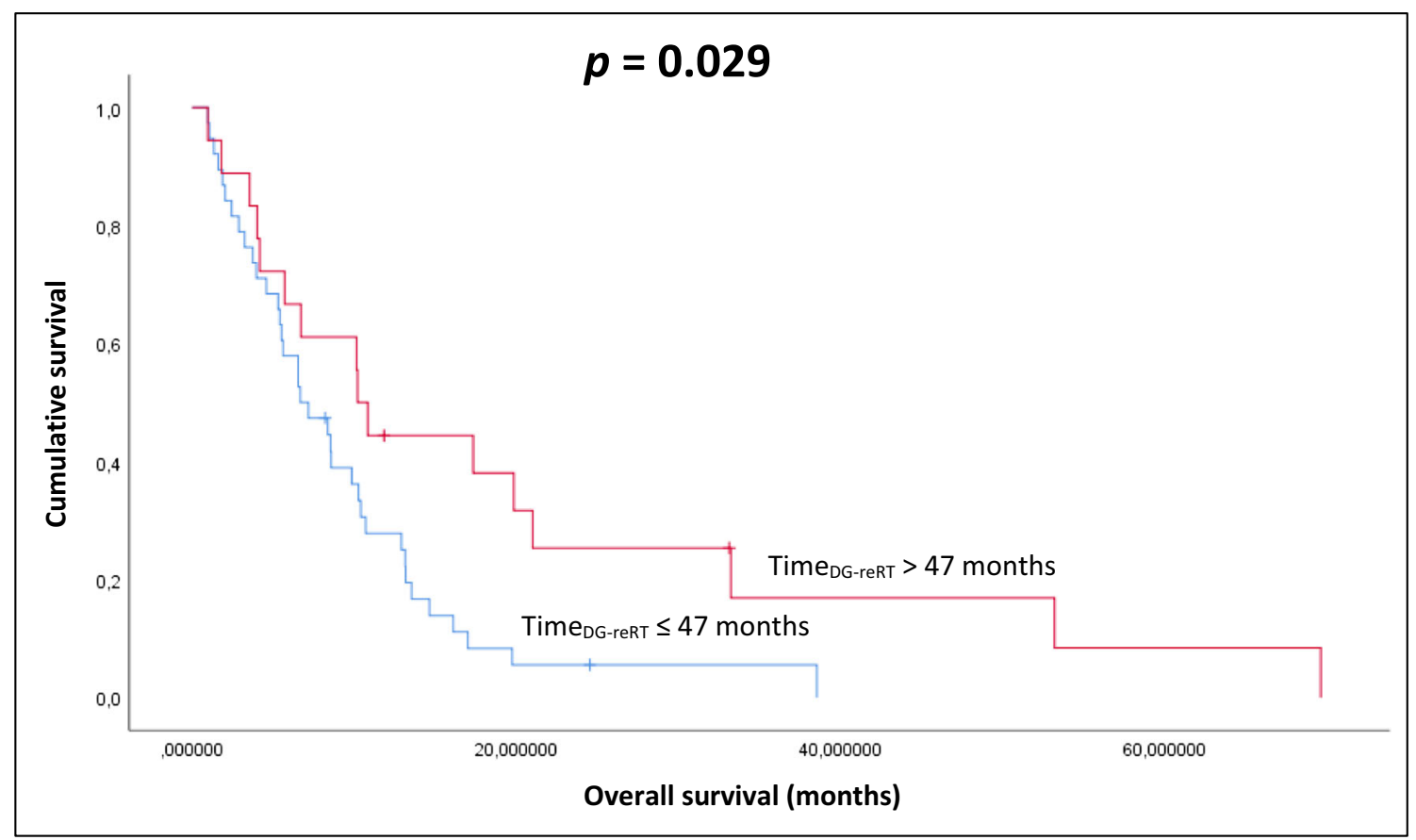

Fig. 3 Kaplan-Meier plot of the correlation between time (DG-reRT) and OS (DG=diagnosis; $\mathrm{p}=0.029$ )

$[17,25,32]$. There are only very few prospective reports on the efficacy of re-RT. Shi and colleagues recently published the late results from RTOG 0525 trial [37]. Patients received BSC only had an mOS of 4.8 months versus the groups treated with re-RT only, chemotherapy only or radiochemotherapy, $8.2,10.5,11.3$ months, respectively [37]. It should be noted that in this study OS was calculated from the first progression and not from the beginning of re-RT, as it is in our present study. Well-defined prognostic factors are established for glial tumours; however, the factors influencing the outcome of reRT are less known. Different factors are considered to influence the efficacy of the survival after re-RT, such as age, performance status, histological grading and the length of the interval between the 1st and the 2nd course of RT [38, 39]. A recent meta-analysis and appraisal summarizes the radiation parameters and outcomes of fractionated re-RT from studies published from 1999 to 2018 [17, 40]. The re-RT was delivered at a median time interval of 12 months (range: 3.5 to 19 months) with dose of 24 to 36 Gy with a daily fractional size of 1.8 to $6 \mathrm{~Gy}$. In our case, $>7$ months passed after the 1 st RT and we applied 1.6 Gy fraction size. The 8.4 months OS of our group is comparable to previous studies, reporting the mOS from re-RT 7.5 to 11 months.

The evaluation of the clinical data in different series of reRT revealed important factors, which may improve the survival, such as KPS $>70 \%$, age $<50$ years, interval $>$ 12 months between the first RT and re-RT, target volume $<$ 20-30 $\mathrm{cm}^{3}$, radiation dose $>30-35 \mathrm{~Gy}$.

In our study, significant predictors for a longer survival after re-RT were the better performance status at re-RT, the longer interval from 1st line treatment to re-RT and lower tumour grade both at diagnosis and at re-RT. The age at reRT proved not to be a prognostic factor, however, the mean age was below 40 years. The tumour size (i.e. GTV) was one of the most significant factors for the prognosis of our patients, whilst the PTV exhibited no significant relationship to the OS. Recurrent tumour volume remained the strongest factor in multivariate analysis $(p=0.038)$. The importance of the interval-factor is in line with former reports of re-RT. It can be assumed that the time of the first relapses after the primary treatment is an indicator of the biological behaviour of the tumour [25, 38, 39]. In our patient group, the median survival according to the histopathological grade was higher than in other reported studies (the median survival is around 5560 months for grade 2 and 18-26 months for grade $3 \mathrm{tu}-$ mours). [41]. It could be explained with the natural patient selection and the younger age (inclusion of paediatric patients).

In our cases, re-challenge of temosolomide was never applied, hence the primary monotherapy part was not limited in time, it was administered up to progression. Therefore, the MGMT promoter hypermethylation had less importance, because the re-irradiation was delivered when all patients developed resistance to TMZ. The MGMT status defined at the initial diagnosis was available for 27 cases, obviously with no significance on survival after re-RT. Other recent proven biological factors, such as ATRX and IDH- mutation were only partially available in our patient group.

Therefore this report is limited by the lack of detailed molecular analysis as well as by the retrospective methodology 
which could result in a selection bias as well as an underreporting of low-grade toxicities. However, the selection bias could be reduced by the homogenous treatment concept for our cohort of patients. Nevertheless, comparison to BSCseries remains to be difficult, and conclusions about survival benefits due to intervention should be drawn with caution. Furthermore, due to the still short survival after re-irradiation, objective long-term responses after re-RT were not possible to assess for all patients.

Due to the therapy, amelioration of neurological signs and KPS were experienced in $58 \%$ of our patients. Control radiological imaging detected stable disease or partial remission in 44 cases $(78.6 \%)$.

Although standards of salvage therapy are not yet defined for recurrent glial tumours, mainly due to paucity of highlevel prospective or randomized controlled studies, re-RT of various technique is an established salvage option for selected patients [42].

\section{Conclusion}

Smaller recurrent tumour size, better PS, longer interval from 1st line treatment to re-RT and lower tumour grade predict better outcome from re-RT. No radiation-associated serious adverse events were observed and the re-RT improved the performance status and neurologic symptoms in the majority of the cases. Re-irradiation with low fraction size in large volume recurrent gliomas proved to be safe and seems to be clinically beneficial in selected patient group.

Author contributions The contributions of the authors to the present study are as follows: (1) the conception and design of the study (Á.D., K.H.), (2) examination, therapy plans and follow-up of the patients (Á.D., Zs.E., A.M., K.H.), (4) radiological assessments (A.Cs.), (5) histopathological diagnostics (Z.R.), (6) neurosurgical treatment (P.B.), (7) radiotherapy planning physicist (E.F.), (8) collection and acquisition of data (Sz.L, L.D., Á.D.), (9) statistical analysis of data (B.D.), (10) interpretation of data (Á.D., K.H.), (11) drafting the article (Á.D., K.H), (12) revising it critically for important intellectual content (J.O., K.H.).

Funding Information Open access funding provided by University of Szeged. The authors confirm independence from the sponsors; the content of the article has not been influenced by the sponsors.

\section{Compliance with Ethical Standards}

Conflict of Interest The authors declare that they have no conflict of interest.

Open Access This article is licensed under a Creative Commons Attribution 4.0 International License, which permits use, sharing, adaptation, distribution and reproduction in any medium or format, as long as you give appropriate credit to the original author(s) and the source, provide a link to the Creative Commons licence, and indicate if changes were made. The images or other third party material in this article are included in the article's Creative Commons licence, unless indicated otherwise in a credit line to the material. If material is not included in the article's Creative Commons licence and your intended use is not permitted by statutory regulation or exceeds the permitted use, you will need to obtain permission directly from the copyright holder. To view a copy of this licence, visit http://creativecommons.org/licenses/by/4.0/.

\section{References}

1. Stupp R, Tonn J-C, Brada M, Pentheroudakis G (2010) High-grade malignant glioma: ESMO Clinical Practice Guidelines for diagnosis, treatment and follow-up. Annal of Oncology 21:v190-v193. https://doi.org/10.1093/annonc/mdq187

2. Dolocek TA, Propp JM, Stroup NE et al (2012) CBTRUS statistical report: primary brain and central nervous system tumors diagnosed in the United States in 2005-2009. Neuro Oncol 14(Suppl 5):1-49

3. Wem PY, Kesari S (2008) Malignant gliomas in adults. New England Med 359:492-507

4. Sherriff J, Tamangani J, Senthil L, Cruickshank G, Spooner D, Jones B et al (2013) Patterns of relapse in glioblastoma multiforme following concomitant chemoradiotherapy with temozolomide. $\mathrm{Br}$ J Radiol 86:20120414

5. Pichlmeier U, Bink A, Schackert G et al (2008) Resection and survival in glioblastoma multiforme: an RTOG recursive partitioning analysis of ALA study patients. Neuro Oncology 10: $1025-1034$

6. Studio R, Hegi ME, Mason WP et al (2009) Effects of radiotherapy with concomitant and adjuvant temozolomide versus radiotherapy alone on survival in glioblastoma in a randomised phase III study: 5 year analysis of the EORTC-NCIC trial. Lancet Oncol 10:459-466

7. Dirks P, Bernstein M, Muller PJ, Tucker WS (1993) The value of re-operation for recurrent glioblastoma. Can J Surg 36:271-275

8. Barbagallo GM, Jenkinson MD, Brodbelt AR (2008) "Recurrent" glioblastoma multiforme, when should we reoperate? Br J Neurosurg 22:452-455

9. Perry JR et al (2010) Phase II trial of continuous dose-intense temozolomide in recurrent malignant glioma: RESCUE study. Clin Oncol 28(12):2051-2057

10. Meryem Aktan M, Koc, Gul Kanyilmaz (2015) Survival following re-irradiation using intensity-modulated radiaton therapy with temozolomide in selected patients with recurrent high grade gliomas. Ann Transl Med 3(20):304. https://doi.org/10.3978/j.issn.23055839.2015.11.29

11. Wang 13X, Guo G, Guan H, Yu Y, Lu J, Jinming Yu (2019) Challenges and potential of PD-1/PD-L1checkpoint blockade immunotherapy for glioblastoma. J Exp Clin Cancer Res 38:87. https://doi.org/10.1186/s13046-019-1085-3

12. Phuphanich S, Wheeler CJ, Rudnick JD, Mazer M, Wang H, Nuño MA et al (2013) Phase I trial of a multi-epitope-pulsed dendritic cell vaccine for patients with newly diagnosed glioblastoma. Cancer Immunol Immunother 62:125-135

13. Liau LM, Ashkan K, Tran DD, Campian JL, Trusheim JE, Cobbs CS et al (2018) First results on survival from a large Phase 3 clinical trial of an autologous dendritic cell vaccine in newly diagnosed glioblastoma. J Transl Med 16:142

14. Jain KK (2018) Critical overview of targeted therapies for glioblastoma. Front Oncol 8:419

15. Taunk NK, Moraes FY, Escorcia FE, Mendez LC, Beal K, Marta GN (2016) External beam re-irradiation, combination chemoradiotherapy, and particle therapy for the treatment of recurrent glioblastoma. Expert Rev Anticancer Ther 16(3):347-358 
16. Amichetti M, Amelio D (2011) A Review of the role of the Reirradiation in recurrent high-grade glioma (HGG). Cancers (Basel) 3:4061-4089

17. Kazmi F, Soon YY, Leong Y, Koh WY, Vellayappan B (2018) Reirradiation for recurrent glioblastoma (GBM) a systematic review and meta-analyis. Journal of Neuro-Oncology 142(1):79-90

18. Minniti G, Scaringi C, De Sanctis V, Lanzetta G, Falco T, Di Stefano D et al (2013) Hypofractionated stereotactic radiotherapy and continuous low- dose temozolomide in patients with recurrent or progressive malignant gliomas. J Neurooncol 111:187-194

19. Grosu AL, Weber WA, Franz M, Stärk S, Piert M, Thamm R et al (2005) Re-irradiation of recurrent high- grade gliomas using amino acid PET (SPECT)/CT/MRI image fusion to determine gross tumor volume for stereotactic fractionated radiotherapy. Int J Radiat Oncol Biol Phys 63:511-519.24

20. Ernst-Stecken A, Ganslandt O, Lambrecht U, Sauer R, Grabenbauer G (2007) Survival and quality of life after hypofractionated stereotactic radiotherapy for recurrent malignant glioma. J Neurooncol 81:287-294.25

21. Wuthrick EJ, Curran WJ, Camphausen K, Lin A, Glass J, Evans J et al (2014) A pilot study of hypofractionated stereotactic radiation therapy and sunitinib in previously irradiated patients with recurrent high- grade glioma. Int J Radiat Oncol Biol Phys 90:369-375.26

22. Patel M, Siddiqui F, Jin J-Y, Mikkelsen T, Rosenblum M, Movsas $\mathrm{B}$ et al (2009) Salvage re-irradiation for recurrent glioblastoma with radiosurgery: radiographic response and improved survival. J Neurooncol 92:185-191

23. Bratengeier K, Oechsner M, Gainey M, Flentje M (2009) Remarks on reporting and recording consistent with the ICRU reference dose. Radiat Oncol 4:44. https://doi.org/10.1186/1748-717X-4-44

24. Lin NU, Lee EQ et al (2015) Response assessment criteria for brain metastases: proposal from the RANO group. Lancet Oncology 16: e270-e278

25. Straube C, Elpula G, Gempt J, Gerhardt J, Bette S, Zimmer C, Schmidt-Graf F, Meyer B, Combs SE (2017) Re-irradiation after gross total resection of recurrent glioblastoma. Strahlenther Onkol. https://doi.org/10.1007/s00066-017-1161-6

26. Fokas E, Wacker U, Gross MW, Henzel M, Encheva E, EngenhartCabillic R (2009) Hypofractionated stereotactic re-irradiation of recurrent glioblastomas. Strahlenther Onkol 185:235-240. https:// doi.org/10.1007/s00066-009-1753-x

27. Niyazi M, Siefert A, Schwarz SB et al (2011) Therapeutic options for recurrent malignant glioma. Radiother Oncol 98:1-14

28. Amelio D, Amichetti M (2012) Radiation therapy for the treatment of recurrent glioblastoma: an overview. Cancers (Basel) 4:257-280

29. Redmond KJ, Mehta M (2015) Stereotactic radiosurgery for glioblastoma. Cureus 7:e413

30. Fogh SE, Andrews DW, Glass J et al (2010) Hypofractionated stereotactic radiation therapy: an effective therapy for recurrent high-grade gliomas. J Clin Oncol 28:3048-3053

31. Chatzikonstantinou G, Zamboglou N, Archavlis E, Strouthos I, Zoga E, Milickovic N, Hilaris B, Baltas D, Rödel C, Tselis N. (2018) CT-guided interstitial HDR-brachytherapy for recurrent glioblastoma multiforme: a 20 -year single-institute experience
Strahlenther Onkol. 194(12):1171-1179. https://doi.org/10.1007/ s00066-018-1358-3

32. Combs SE, Niyazi M, Adeberg S, Bougatf N, Kaul D et al (2018) Re-irradiation of recurrent gliomas: pooled analysis and validation of an established prognostic score-report of the Radiation Oncology Group (ROG) of the German Cancer Consortium (DKTK). Cancer Med 7(5): 1742-1749

33. Krauze AV, Peters C, Cheng J et al (2017) Re-irradiation for recurrent glioma- the NCI experience in tumor control, OAR toxicity and proposal of a novel prognostic scoring system. Radiat Oncol Lond Engl 12(1):191. https://doi.org/10.1186/s13014-017-0930-9

34. Zwirner K, Paulsen F, Schittenhelm J et al (2017) Prognostic parameters and outcome after re-irradiation for progressive glioblastoma. Acta Neurol Scand 136:239-245

35. Klobukowski L, Falkov A, Chelimo C et al (2018) A retrospective review of re-irradiating patients' recurrent high-grade gliomas. Clin Oncol (R Coll Radiol) 30:563-570

36. Post CCB, Kramer MCA, Smid EJ et al (2019) Patterns of reirradiation for recurrent gliomas and validation of a prognostic score. Radiother Oncol 130:156-163

37. Shi W, Scannell Bryan M, Gilbert MR, Mehta MP, Blumenthal DT, Brown PD, Valeinis E, Hopkins K, Souhami L, Andrews DW, Tzuk-Shina T, Howard SP, Youssef EF, Lessard N, Dignam JJ, Werner-Wasik M (2017) Investigating the effect of reirradiation or systemic therapy in patients with glioblastoma after tumor progression: a secondary analysis of NRG oncology/radiation therapy oncology group trial 0525. Int J Radiat Oncol Biol Phys 100:38-44. https://doi.org/10.1016/j.ijrobp.2017.08.038

38. Combs SE, Edler L, Rausch R, Welzel T, Wick W, Debus J (2013) Generation and validation of a prognostic score to predict outcome after re-irradiation of recurrent glioma. Acta Oncol 52:147-152. https://doi.org/10.3109/0284186X.2012.692882

39. Kessel KA, Hesse J, Straube C, Zimmer C, Schmidt-Graf F, Schlegel J, Meyer B, Combs SE (2017) Modification and optimization of an established prognostic score after re-irradiation of recurrent glioma. PLoS ONE 12:e0180457. https://doi.org/10.1371/ journal.pone. 0180457

40. Kim IH (2019) Appraisal of re-irradiation for the recurrent glioblastoma in the era of MGMT promotor methylation. Radiat Oncol $\mathrm{J}$ 37(1):1-12. https://doi.org/10.3857/roj.2019.00171T

41. Tunthanathip T, Ratanalert S, Sae-heng S, Oearsakul T, Sakaruncchai I, Kaewborisutsakul A, Chotsampancharoen T, Intusoma U, Kitkhuandee A, Vaniyapong T (2020) Prognostic Factors and Nomogram Predicting Survival in Diffuse Astrocytoma. J Neurosci Rural Pract 11(1):135-143. https://doi. org/10.1055/s-0039-3403446

42. Guyotat J, Signorelli F, Frappaz D, Madarassy G, Ricci AC, Bret P (2000) Is re-operation for recurrence of glioblastoma justified? Oncol Rep 7:899-904

Publisher's Note Springer Nature remains neutral with regard to jurisdictional claims in published maps and institutional affiliations. 